\title{
Residual Hearing Preservation with the Evo® Cochlear Implant Electrode Array: Preliminary Results
}

\author{
Ricardo Ferreira Bento ${ }^{1}$ Fabiana Danieli ${ }^{2}$ Ana Tereza de Matos Magalhães ${ }^{3} \quad$ Dan Gnansia ${ }^{4}$ Michel Hoen $^{4}$ \\ ${ }^{1}$ Department of Otolaryngology, Universidade de São Paulo, \\ São Paulo, Brazil \\ ${ }^{2}$ Clinical Department, Oticon Medical, São Paulo, Brazil \\ ${ }^{3}$ Department of Audiology, Universidade de São Paulo, São Paulo, \\ Address for correspondence Michel Hoen, PhD, Department of \\ Scientific and Clinical Research, Oticon Medical, 2720 Chemin St., \\ Bernard Porte 14, Vallauris 06220, France \\ (e-mail: mhoe@oticonmedical.com).
} Brazil

${ }^{4}$ Department of Scientific and Clinical Research, Oticon Medical,

Vallauris, France

Int Arch Otorhinolaryngol 2016;20:353-358.

\begin{abstract}
Keywords

- cochlear implantation

- cochlear round window

- residual hearing

- hearing preservation

- dexamethasone

Introduction The preservation of residual hearing is currently an important challenge for cochlear implant surgeries. Indeed, if patients exhibit functional hearing after cochlear implantation, they can benefit from the combination of acoustical stimulation, usually in the low-frequencies and electrical stimulation in the high-frequencies. This combined mode of stimulation has proven to be beneficial both in terms of speech perception and of sound quality. Finding the right procedures for conducting softsurgeries and designing electrode arrays dedicated to hearing preservation is an open issue.

Objective The objective of this study is to evaluate the combination of a soft-surgery procedure implicating round-window insertion and the use of dexamethasone and hyaluronic acid during surgery, with the use of a specifically designed straight soft electrode array, on hearing preservation in patients with functional hearing in the low frequencies.

Methods This pre-clinical trial was conducted on seven patients with residual hearing in the low frequencies. The surgical method used employed a round window insertion and the use of topical dexamethasone.

Results The soft-surgery protocol could be successfully followed in five patients. In this group, the average hearing threshold shift compared with pre-operative values was of $18.7+/-16.1 \mathrm{~dB} \mathrm{HL}$ up to $500 \mathrm{~Hz}$ and $15.7+/-15.1$ up to $1 \mathrm{kHz}$, demonstrating satisfying levels of hearing preservation.

Conclusion We were able to demonstrate the possibility of preserving residual hearing in most of the patients using the EVO electrode. Significant residual hearing preservation levels were was obtained when a soft surgical approach involving round window insertion, dexamethasone and hyaluronic use during the surgery.
\end{abstract}

received

October 6, 2015

accepted

November 15, 2015

published online

February 16, 2016
DOI http://dx.doi.org/

10.1055/s-0036-1572530. ISSN 1809-9777.
Copyright $(2016$ by Thieme Publicações License terms

Ltda, Rio de Janeiro, Brazil 


\section{Introduction}

Cochlear implants (CI) nowadays constitute a method of choice for the rehabilitation of hearing function in patients with severeto-profound hearing loss and congenital deafness. Thanks to proven benefits in terms of quality of life and speech and communication, $\mathrm{Cl}$ indication was progressively extended to patients presenting significant levels of functional residual hearing. Provided cochlear implantation can preserve this residual hearing, these patients can benefit from the combination of electrical stimulation provided by the $\mathrm{CI}$ over mid- to high frequencies and of amplified acoustic stimulation over the low-frequencies. This stimulation mode was shown to improve speech understanding in various listening situations. ${ }^{1,2}$ These advantages have highlighted the importance of preserving residual hearing during $\mathrm{Cl}$ surgery.

Recent developments in this area followed mainly three lines of research. The first series of improvements concerned the surgery itself with the refining of soft-surgical approaches, seeking to minimize intracochlear trauma due to the opening of the internal ear and the insertion of the electrode array. In this context, the round-window (RW) surgical approach is often thought to be less traumatic than the cochleostomy. With the RW approach, the entry into the cochlea happens through a membrane, not requiring added bone drilling and can thus potentially lead to less trauma., Therefore, the RW approach today constitutes the favored approach for soft-surgeries and is still widely preferred over cochleostomy. The second line of developments concerned the use of pharmacological support to diminish the acute insertion trauma as well as postimplantation inflammation, and lubricating substances, to diminish the mechanical trauma caused during the electrode insertion. Dexamethasone was proven to be efficient under systemic administration. The injection of intravenous dexamethasone one hour prior to the surgery lead to reduced surgery-induced hearing loss. ${ }^{5}$ Preoperative local administration of steroids directly on the RW was shown to significantly reduce post-operative thresholds shifts. ${ }^{6}$ In addition to steroids, the use of lubricating fluids or gels applied onto the electrode array, such as Hyaluronic acid was shown to reduce friction forces during electrode insertion, potentially limiting the insertion trauma. ${ }^{7,8}$ Finally, the combination of both steroids and hyaluronic acid was sometimes more efficient than the use of either solution alone. ${ }^{9}$ The last line of research developed in this context was the improvement and specific design of electrode arrays optimized for less traumatic insertions. Shorter electrode arrays, meant to cover only the high-frequency regions of the cochlea were developed, but presented very limited interest because of the frequent progressive nature of the hearing-loss affecting patients and the high risk of reimplantation associated. Moreover, residual hearing preservation was shown to be possible with long electrodes, provided they have a soft surface and a limited tip diameter. ${ }^{10,11}$

Despite these results, the efficiency of combining softsurgical approaches with specific pharmacological treatment procedures to prevent loss of residual hearing using long electrode arrays can still be optimized and specific protocols should be tested to develop clear guidelines. The objective of the present study was to evaluate the combination of a softsurgery procedure implicating round-window insertion and the use of dexamethasone and hyaluronic acid during surgery, with the use of a specifically designed soft electrode array (EVO, Oticon Medical, Vallauris, France), on hearing preservation in a group of $\mathrm{Cl}$ candidates with residual hearing in the low frequencies.

\section{Method}

\section{Participants}

Seven participants from the Department of Otolaryngology of the Universidade de São Paulo (São Paulo, Brazil) were included in this preliminary clinical study. Participants were all post-lingual deaf adults, aged 23 to 83 years, with an average age of $52.43^{+} / .20 .85$ years. Patient's demographic details are provided in -Table 1. All but one patient had a history of progressive hearing loss from unknown etiology. The last patient had a sudden ototoxic loss. Presurgical imaging revealed no cochlear abnormalities or ossifications of the internal ear in any patient. All presented with profound hearing loss thresholds starting from $1000 \mathrm{~Hz}$, with averaged pure-tone thresholds between 1 and $8 \mathrm{kHz}$ of $119.7^{+} /$ $12.8 \mathrm{~dB}$ HL. See $\boldsymbol{-}$ Fig. 1 for detailed pure-tone audiometry. Patients were included in the study because they presented residual hearing over the low frequencies, with averaged pure-tone thresholds at $750 \mathrm{~Hz}=86.1^{+} / .14 .85 \mathrm{~dB} \mathrm{HL}$; $500 \mathrm{~Hz}=67.9^{+} / .12 .5 \mathrm{~dB} \mathrm{HL} ; 250 \mathrm{~Hz}=46.4^{+} / .10 .7 \mathrm{~dB} \mathrm{HL}$ and $125 \mathrm{~Hz}=38.6^{+} / .10 .7 \mathrm{~dB} \mathrm{HL}$ ). Despite these favorable low-frequency thresholds, all patients had limited benefit of hearing aids (Sentence recognition scores lower than 50\% with amplification), and were thus candidates for cochlear implantation and received an Oticon Medical Neurelec (Vallauris, France) CI system (Digisonic SP). If residual hearing was preserved, these patients would be candidates for

Table 1 Patients' demographics

\begin{tabular}{|l|l|l|l|l|}
\hline $\begin{array}{l}\text { Subject } \\
\text { id }\end{array}$ & Gender & $\begin{array}{l}\text { Age at } \\
\text { Implantation } \\
\text { (years) }\end{array}$ & $\begin{array}{l}\text { Implanted } \\
\text { Side }\end{array}$ & Etiology \\
\hline P1 & F & 83 & Left & $\begin{array}{l}\text { Progr. } \\
\text { Unknown }\end{array}$ \\
\hline P2 & F & 41 & Left & $\begin{array}{l}\text { Progr. } \\
\text { Unknown }\end{array}$ \\
\hline P3 & $\mathrm{F}$ & 72 & Right & $\begin{array}{l}\text { Progr. } \\
\text { Unknown }\end{array}$ \\
\hline P4 & $\mathrm{F}$ & 49 & Left & $\begin{array}{l}\text { Progr. } \\
\text { Unknown }\end{array}$ \\
\hline P5 & $\mathrm{F}$ & 23 & Left & $\begin{array}{l}\text { Progr. } \\
\text { Unknown }\end{array}$ \\
\hline P6 & M & 38 & Right & $\begin{array}{l}\text { Sudden } \\
\text { Ototoxic }\end{array}$ \\
\hline P7 & F & 61 & Left & $\begin{array}{l}\text { Progr. } \\
\text { Unknown }\end{array}$ \\
\hline
\end{tabular}

Abbreviations: id, identification; F, female; M, male; Progr, progressive. 

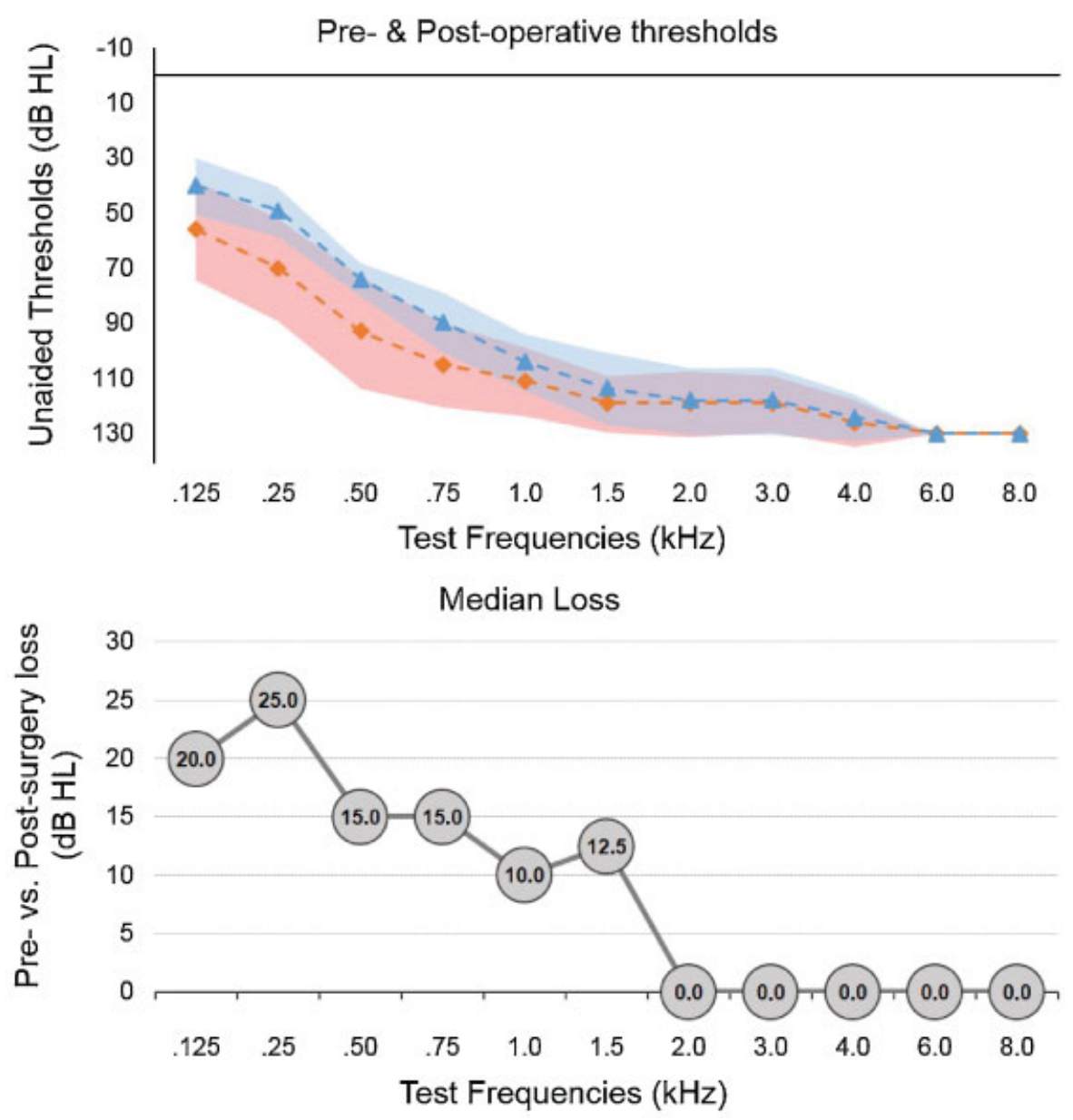

Fig. 1 Top panel: Averaged free-field warble-tone thresholds measured pre- (blue) and post-operatively (orange) in the five patients who had successful soft-surgery with the Evo ${ }^{\circledR}$ electrode array. The shaded areas represent the standard deviation of mean. Bottom-panel: graphical representation of the median loss across frequencies for the same patients.

combined electric and acoustic stimulation using the Zebra (Oticon Medical, Vallauris, France) speech processor. ${ }^{12}$ Before inclusion, volunteers were fully informed about the goals and procedures of the study and provided written consent. This study was performed in accordance with the Declaration of Helsinki, and was approved by the Ethics Committee for the Analysis of Research projects of the Universidade de São Paulo (São Paulo, Brazil).

\section{Hearing Preservation Soft-Surgery Protocol and Electrode Array}

The soft surgical procedure employed a classical trans-mastoid approach and a round-window insertion of the electrode array. ${ }^{9}$ Pre-operatively, patients received a weight-adjusted intravenous dose of hydrocortisone $(4 \mathrm{mg} / \mathrm{kg}$ ) and a single dose of preventive antibiotic treatment (cefazolin, $1 \mathrm{~g}$ ), before intubation and general anesthesia. The surgeon then performed a wide posterior tympanotomy via facial recess, allowing exposure of the round window membrane. The middle ear cavity was then filled with a dexamethasone solution $(4 \mathrm{mg} / \mathrm{ml})$, while the receiver was placed in the subperiosteal pocket and secured with two titanium screws, without drilling a bony well. ${ }^{13,14}$ The steroid solution was then removed, the anterior border of the round window membrane was delicately perforated and no suctioning of the perilymph was performed. Hyaluronic acid (Provisc, Alcon Laboratories Inc., Puurs, Belgium) was then placed over the membrane and along the electrode array, before slow insertion of the electrode. After insertion, the electrode insertion site was sealed using a small collar of temporalis fascia positioned around the electrode array and the middle ear cavity was again filled with dexamethasone $(4 \mathrm{mg} / \mathrm{ml})$. If the soft-surgery was compromised (difficult insertion of electrode array), the procedure would be abandoned and a cochleostomy performed to warranty the best insertion ratio and the best cochlear implant outcomes for the patient despite the risk of not preserving residual hearing.

\section{The EVO® Electrode Array}

The EVO ${ }^{\circledR}$ electrode array (Oticon Medical, Vallauris, France) was especially designed to respond to the constraints of soft surgeries for hearing preservation. It is a long ( $24 \mathrm{~mm})$, thin (proximal diameter $=0.5 \mathrm{~mm}$; distal diameter $=0.4 \mathrm{~mm}$ ), and flexible device, with a smooth surface silicone array carrying 20 micro-machined titanium-iridium electrodes (-Fig. 2). This specific design demonstrated low insertion forces compared with classical electrode array designs, ${ }^{15}$ and is associated to low levels of intracochlear traumas. ${ }^{16}$ 


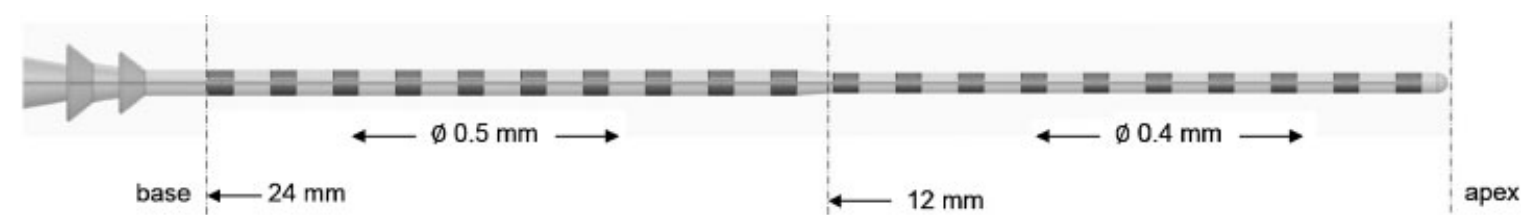

Fig. 2 The EVO® electrode-array for residual hearing preservation. The electrode array was designed for maximized hearing preservation with a smooth surface and a soft, less traumatic tip.

\section{Evaluation of Residual Hearing Preservation}

To evaluate residual hearing preservation, we evaluated pre- and post-surgical pure-tone audiometry using a calibrated clinical AC33 audiometer (Interactoustics, Assens, Denmark), in a double-walled sound-booth. We measured warble-tone thresholds under free-field listening conditions, using only the cochlear implant system (contralateral ear plugged). Post-operative thresholds were measured on average $3.14^{+} / .1 .5$ months after surgery.

\section{Results}

\section{Surgeries}

The round-window soft surgery approach was possible only in five out of seven patients (P1; P4-P7). Unfortunately, electrode insertion through the RW appeared to be difficult and remained partial in two patients (P2 \& P3). These two patients required a cochleostomy to obtain a more favorable insertion angle and ensure a fuller insertion. Therefore, we will first report separate results for the 5 patients who had a successful soft-surgery and round window insertion, which represent our best case scenario. We will then report on the complete group by including the two patients who had a cochleostomy.

\section{Hearing Preservation with Soft-Surgery and Round Window Insertion}

The different thresholds measured pre- and post-surgery in the group of patients who had successful soft-surgery are represented in - Fig. 1. In this group, the average hearing threshold shift compared with pre-operative values was of $18.7^{+} / .16 .1 \mathrm{~dB}$ HL up to $500 \mathrm{~Hz}$ and $15.7^{+} / .15 .1$ up to $1 \mathrm{kHz}$ ( - Fig. 1-Top panel). The observed median loss at $500 \mathrm{~Hz}$ was $15 \mathrm{~dB}$ HL (-Fig. 1-Bottom panel). The averaged residual thresholds after surgery in this group of patients were 73 +/. $24 \mathrm{~dB} \mathrm{HL}$ up to $500 \mathrm{~Hz}$ and $87^{+} / .26 .7 \mathrm{up}$ to $1 \mathrm{kHz}$.

However, these group's results hide a relatively important interindividual variability as can shown in -Fig. $\mathbf{3}$, showing the pre- versus post-surgery thresholds diagram at $500 \mathrm{~Hz}$. Among the five patients, 3 (P1, P5 \& P7) had post-operative thresholds in the $+10 \mathrm{~dB}$ HL range and one more (P4) was between +10 and $+20 \mathrm{~dB}$ HL. One patient (P6) showed an important threshold shift of $+40 \mathrm{~dB} \mathrm{HL}$ at $500 \mathrm{~Hz}$.

\section{Complete Group Analysis}

When considering the data from the complete patients group, results showed that the two patients (P2 \& P3, triangles on -Fig. 3), who could not have a full-insertion through

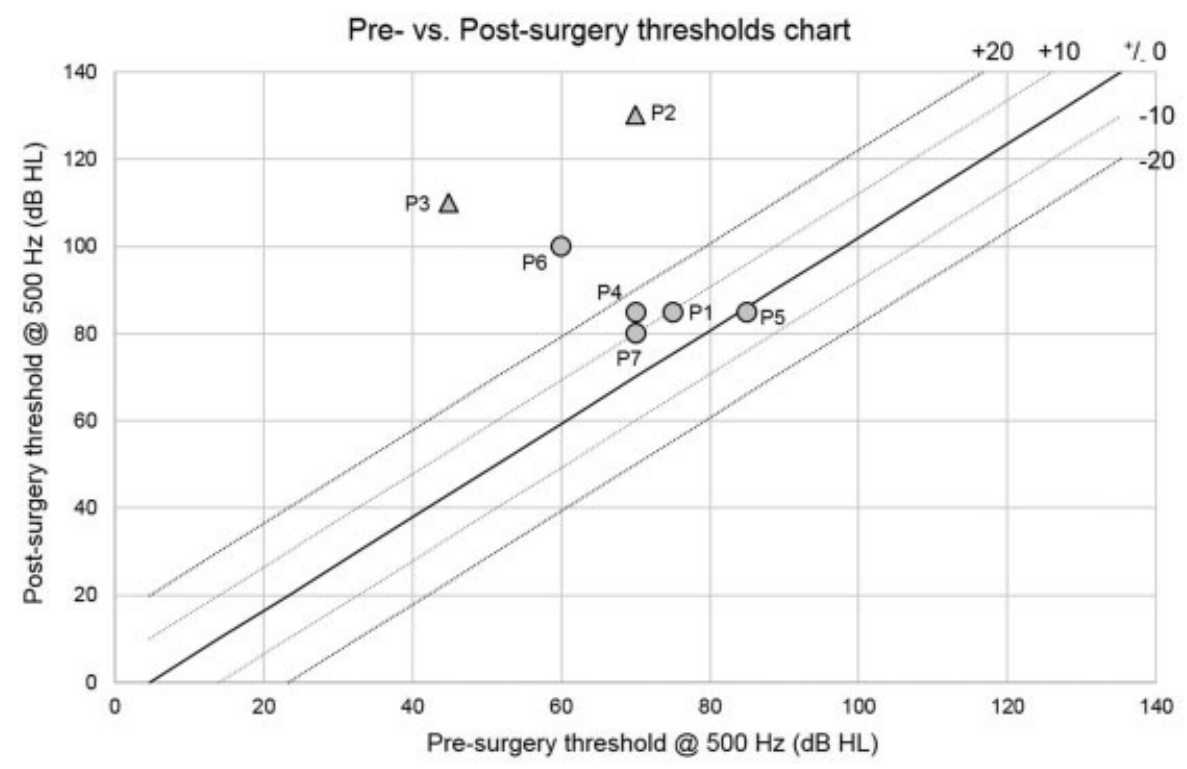

Fig. 3 Pre- versus post-surgery input/output chart. For each individual patient included in the study, pre- ( $x$-axis) and post-operative ( $y$-axis) thresholds are plotted on the same graph. The diagonal (black line) of this graph represents the no-difference line the dotted lines represent the ${ }^{+} / .10 \mathrm{~dB} \mathrm{HL}$ (light gray) or ${ }^{+} / .20 \mathrm{~dB}$ HL difference lines (dark gray). Circles represent patients from the round-window insertion group and triangles represent patients $\mathrm{P} 2$ and $\mathrm{P} 3$, who had to undergo cochleostomy because of difficult insertion (partial ossification). 
the round-window showed worst results than the patients from the round-window insertion group, with individual threshold increases at $500 \mathrm{~Hz}$ of respectively 40 and $65 \mathrm{~dB}$ HL. However, even for these two patients, some residual hearing could be preserved as their averaged thresholds below $500 \mathrm{~Hz}$ were of $81.7^{+} /$- 19.7. Including these two patients, the group-averaged hearing threshold shift compared with pre-operative values was of $24.5^{+} / .18 .2 \mathrm{~dB}$ HL up to $500 \mathrm{~Hz}$ and $21.8^{+} / .19 .2$ up to $1 \mathrm{kHz}$.

\section{Discussion}

This preliminary clinical study demonstrated the possibility of preserving residual hearing using the $24 \mathrm{~mm}$ long EVO ${ }^{\circledR}$ soft electrode array combined with a soft-surgery protocol including the usage of dexamethasone and hyaluronic acid. ${ }^{9}$ The results, however, also highlighted potential caveats of this approach, regarding the selection of candidate patients and the surgical approach employed.

In a first sub-group of five patients, thresholds compatible with the use of combined electric and acoustic stimulation could be achieved. Although the number of patients included in the present study do not allow us to generalize from our current observations, these preliminary observations are very promising and would encourage the conducting of larger scale, multi-centric clinical trials including larger numbers of patients. In 3 out of 5 patients (60\%), who had successful softsurgery with round-window insertion, the hearing preservation was complete, with a post-surgery loss at $500 \mathrm{~Hz}$ of $\leq 10 \mathrm{~dB}$ and moderate (loss of $11-20 \mathrm{~dB}$ ) in one other patient (20\%) and one presented only partial hearing preservation (loss of $\leq 40 \mathrm{~dB}$ ), bringing the total count of partial preservations to $100 \%$. When including the two patients who could not have soft-surgery and who had to have a cochleostomy after several attempts of RW insertion, the number is $71.4 \%$ of successful hearing preservation over the sample. Our preliminary observations are well in line with data reported in the literature regarding hearing preservation. Reported distributions in the literature vary with the study and are in about the same range: $45.2 \%$ of complete preservation and $90.3 \%$ of partial loss in 31 children, mean threshold shift of $18.5 \mathrm{~dB}$ HL with standard electrode arrays and full-length insertions ${ }^{17} ; 71-86 \%$ hearing preservation using the Nucleus 24 Contour Advance electrode. ${ }^{18}$ In a recent study comparing hearing preservation with the Hybrid-L24 and the CI 422 from Cochlear, Jurawitz and colleagues ${ }^{19}$ reported $54.6 \%$ and $49.0 \%$ of subjects showed a mean threshold shift $<15 \mathrm{~dB}$. Another aspect which would be interesting to monitor is the evolution over time of the hearing preservation scores, several authors having shown that sometimes scores obtained pre-operatively decrease during the following months. ${ }^{20,21}$

Our data also suggest that hearing preservation can be achieved with a straight electrode with full insertion $(21 \mathrm{~mm})$, provided a soft-surgical procedure can be achieved. The round-window surgical approach led to good hearing preservation results in our case, however, we cannot be conclusive regarding the difference between the RW and a cochleostomy approach, because of the small numbers of patients included in the present study and because a cochleostomy was performed only if the RW approach could not be conducted properly. Former work in this domain have shown that both approaches could lead to satisfying hearing preservation levels provided both are conducted appropriately. In a systematic literature review including 170 patients, Havenith and colleagues, ${ }^{22}$ could not find clear evidence for a superiority of the RW approach compared with cochleostomy. Other authors reported that similar levels of lowfrequency hearing preservation could be achieved using straight narrow electrode inserted with either approaches. ${ }^{23-25}$ One possible difference contrasting both approaches is the relatively important anatomical variability of the round window itself, which could compromise insertion in certain cases and would make the cochleostomy a better option. ${ }^{26}$ Extensive studies including more patients and looking at the detailed relationship between ore-operative anatomical characteristics of the RW region and how these should constrain the choice of the surgical approach are necessary.

Our study also provides evidence for the use of steroids such as Dexamethasone, combined with Hyaluronic acid to ease atraumatic electrode insertion. ${ }^{9}$ These observations constitute arguments in favor of improving the way drugs can be delivered to the inner ear to favor long-term residual hearing preservation in patients candidates for combined electrical and acoustical stimulation. One option in this domain would be to develop drug-eluting electrode arrays, made with dexamethasone loaded silicones, which could deliver steroids to the inner ear over longer post-surgery periods. ${ }^{27,28}$ Further work will be dedicated to the amelioration of these treatment options to maximize hair-cell survival rate after $\mathrm{Cl}$ surgery. The preservation of residual hearing in $\mathrm{CI}$ surgeries will allow patients to benefit from combined acoustical and electrical stimulation, allowing for better functional outcomes compared with electrical stimulation alone, in particular concerning speech perception and speech-in-noise comprehension. This combined mode of stimulation was indeed shown to increase the perception of low-frequency cues (F0, f1) in speech. F0 was shown to play a crucial role in voice separation and auditory stream segregation, ${ }^{29}$ and lowfrequency cues are also crucial for phoneme categorization in normal listeners ${ }^{30}$ and $\mathrm{CI}$ users. ${ }^{31}$ Follow-up experiments will therefore measure the speech perception and speech in noise intelligibility outcomes in the patients who had successful residual hearing preservation, using a sound processor designed to deliver both acoustical and electrical stimulations. Altogether, the benefits of soft surgery techniques and the ameliorations observed regarding the levels of residual hearing preservation consecutively to a cochlear implantation are progressively broadening the indications of cochlear implants.

\section{Conclusions}

In this preliminary clinical study involving seven $\mathrm{CI}$ patients, we could demonstrate the possibility of preserving residual 
hearing in most of the patients using a specifically designed straight soft electrode (EVO, Oticon Medical, Vallauris, France). Residual hearing preservation was obtained in those patients in which a soft surgical approach involving round window insertion, pre-surgical dexamethasone, and topical dexamethasone use during the surgery, as well as the coating of the electrode array using hyaluronic acid. In certain cases, due to anatomical variations in the round widow region and to partial intracochlear ossification, this surgery was not possible and hearing preservation could not be achieved. These results shed light on the importance of the surgical approach combined to electrode array specificities for successful hearing preservation in $\mathrm{CI}$ patients.

\section{References}

1 Gantz BJ, Turner C, Gfeller KE, Lowder MW. Preservation of hearing in cochlear implant surgery: advantages of combined electrical and acoustical speech processing. Laryngoscope 2005;115(5):796-802

2 Gifford RH, Dorman MF, Skarzynski H, et al. Cochlear implantation with hearing preservation yields significant benefit for speech recognition in complex listening environments. Ear Hear 2013; 34(4):413-425

3 Richard C, Fayad JN, Doherty J, Linthicum FH Jr. Round window versus cochleostomy technique in cochlear implantation: histologic findings. Otol Neurotol 2012;33(7):1181-1187

4 Nordfalk KF, Rasmussen K, Bunne M, Jablonski GE. Deep round window insertion versus standard approach in cochlear implant surgery. Eur Arch Otorhinolaryngol 2014

5 Kuthubutheen J, Coates H, Rowsell C, Nedzelski J, Chen JM, Lin V. The role of extended preoperative steroids in hearing preservation cochlear implantation. Hear Res 2015;327:257-264

6 Chang A, Eastwood H, Sly D, James D, Richardson R, O'Leary S. Factors influencing the efficacy of round window dexamethasone protection of residual hearing post-cochlear implant surgery. Hear Res 2009;255(1-2):67-72

7 Laszig R, Ridder GJ, Fradis M. Intracochlear insertion of electrodes using hyaluronic acid in cochlear implant surgery. J Laryngol Otol 2002;116(5):371-372

8 Miroir M, Nguyen Y, Kazmitcheff G, Ferrary E, Sterkers O, Grayeli $\mathrm{AB}$. Friction force measurement during cochlear implant insertion: application to a force-controlled insertion tool design. Otol Neurotol 2012;33(6):1092-1100

9 Ramos BF, Tsuji RK, Bento RF, et al. Hearing preservation using topical dexamethasone alone and associated with hyaluronic acid in cochlear implantation. Acta Otolaryngol 2015;135(5):473-477

10 Nguyen Y, Mosnier I, Borel S, et al. Evolution of electrode array diameter for hearing preservation in cochlear implantation. Acta Otolaryngol 2013;133(2):116-122

11 Brown KD, Melton MF, Shonfield H, Kraskin M, Wolf J. Preserved low-frequency hearing following $20-\mathrm{mm}$ cochlear implantation. Otol Neurotol 2015;36(2):240-243

12 Vaerenberg B, Péan V, Lesbros G, et al. Combined electric and acoustic hearing performance with Zebra ${ }^{\circledR}$ speech processor: speech reception, place, and temporal coding evaluation. Cochlear Implants Int 2013;14(3):150-157

13 Guevara N, Sterkers O, Bébéar JP, et al. Multicenter evaluation of the digisonic SP cochlear implant fixation system with titanium screws in 156 patients. Ann Otol Rhinol Laryngol 2010;119(8):501-505
14 Carvalho GM, Guimarães AC, Danieli F, et al. Evaluation of the Digisonic ${ }^{\circledR}$ SP cochlear implant: patient outcomes and fixation system with titanium screws. Braz J Otorhinolaryngol 2012;78(6): $56-62$

15 Nguyen Y, Miroir M, Kazmitcheff G, et al. Cochlear implant insertion forces in microdissected human cochlea to evaluate a prototype array. Audiol Neurootol 2012;17(5):290-298

16 Martins GdeS, Brito Neto RV, Tsuji RK, Gebrim EM, Bento RF. Evaluation of intracochlear trauma caused by insertion of cochlear implant electrode arrays through different quadrants of the round window. Biomed Res Int 2015;2015:236364

17 Brown RF, Hullar TE, Cadieux JH, Chole RA. Residual hearing preservation after pediatric cochlear implantation. Otol Neurotol 2010;31(8):1221-1226

18 Garcia-Ibanez L, Macias AR, Morera C, et al. An evaluation of the preservation of residual hearing with the Nucleus Contour Advance electrode. Acta Otolaryngol 2009;129(6):651-664

19 Jurawitz MC, Büchner A, Harpel T, et al. Hearing preservation outcomes with different cochlear implant electrodes: Nucleus ${ }^{\circledR}$ Hybrid $^{\mathrm{TM}}$-L24 and Nucleus Freedom ${ }^{\mathrm{TM}}$ CI422. Audiol Neurootol 2014;19(5):293-309

20 Santa Maria PL, Domville-Lewis C, Sucher CM, Chester-Browne R, Atlas MD. Hearing preservation surgery for cochlear implantation -hearing and quality of life after 2 years. Otol Neurotol 2013; 34(3):526-531

21 Bruce IA, Felton M, Lockley M, et al. Hearing preservation cochlear implantation in adolescents. Otol Neurotol 2014;35(9): 1552-1559

22 Havenith S, Lammers MJ, Tange RA, et al. Hearing preservation surgery: cochleostomy or round window approach? A systematic review. Otol Neurotol 2013;34(4):667-674

23 Adunka OF, Dillon MT, Adunka MC, King ER, Pillsbury HC, Buchman CA. Cochleostomy versus round window insertions: influence on functional outcomes in electric-acoustic stimulation of the auditory system. Otol Neurotol 2014;35(4):613-618

24 Hassepass F, Aschendorff A, Bulla S, et al. Radiologic Results and Hearing Preservation With a Straight Narrow Electrode via Round Window Versus Cochleostomy Approach at Initial Activation. Otol Neurotol 2015;36(6):993-1000

25 Sun $\mathrm{CH}, \mathrm{Hsu} \mathrm{CJ}$, Chen PR, Wu HP. Residual hearing preservation after cochlear implantation via round window or cochleostomy approach. Laryngoscope 2015;125(7):1715-1719

26 Zhou L, Friedmann DR, Treaba C, Peng R, Roland JT Jr. Does cochleostomy location influence electrode trajectory and intracochlear trauma? Laryngoscope 2015;125(4):966-971

27 Nguyen Y, Bernardeschi D, Kazmitcheff G, et al. Effect of embedded dexamethasone in cochlear implant array on insertion forces in an artificial model of scala tympani. Otol Neurotol 2015;36(2): 354-358

28 Sircoglou J, Gehrke M, Tardivel M, Siepmann F, Siepmann J, Vincent C. Trans-Oval-Window Implants, A New Approach for Drug Delivery to the Inner Ear: Extended Dexamethasone Release From Silicone-based Implants. Otol Neurotol 2015;36(9):1572-1579

29 Bronkhorst AW. The cocktail-party problem revisited: early processing and selection of multi-talker speech. Atten Percept Psychophys 2015;77(5):1465-1487

30 Varnet L, Knoblauch K, Serniclaes W, Meunier F, Hoen M. A psychophysical imaging method evidencing auditory cue extraction during speech perception: a group analysis of auditory classification images. PLoS ONE 2015;10(3):e0118009

31 Goldsworthy RL. Correlations Between Pitch and Phoneme Perception in Cochlear Implant Users and Their Normal Hearing Peers. J Assoc Res Otolaryngol 2015;16(6):797-809 